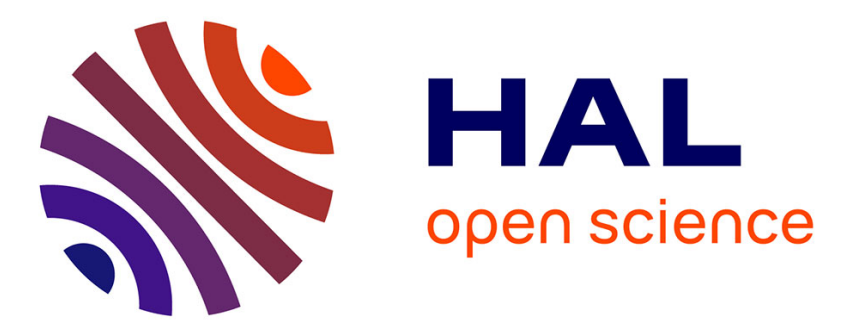

\title{
MR prior based automatic segmentation of the prostate in TRUS images for MR/TRUS data fusion
}

Sébastien Martin, Michael Baumann, Vincent Daanen, Jocelyne Troccaz

\section{To cite this version:}

Sébastien Martin, Michael Baumann, Vincent Daanen, Jocelyne Troccaz. MR prior based automatic segmentation of the prostate in TRUS images for MR/TRUS data fusion. IEEE International Symposium on Biomedical Imaging, ISBI'2010, Apr 2010, Rotterdam, Netherlands. pp.640-643. hal00477748

\section{HAL Id: hal-00477748 \\ https://hal.science/hal-00477748}

Submitted on 30 Apr 2010

HAL is a multi-disciplinary open access archive for the deposit and dissemination of scientific research documents, whether they are published or not. The documents may come from teaching and research institutions in France or abroad, or from public or private research centers.
L'archive ouverte pluridisciplinaire HAL, est destinée au dépôt et à la diffusion de documents scientifiques de niveau recherche, publiés ou non, émanant des établissements d'enseignement et de recherche français ou étrangers, des laboratoires publics ou privés. 


\title{
MR PRIOR BASED AUTOMATIC SEGMENTATION OF THE PROSTATE IN TRUS IMAGES FOR MR/TRUS DATA FUSION
}

\author{
Sébastien Martin ${ }^{1}$, Michael Baumann ${ }^{1,2}$, Vincent Daanen ${ }^{2}$, Jocelyne Troccaz $^{1 *}$ \\ ${ }^{1}$ Université J.Fourier, TIMC laboratory, Grenoble, France; CNRS, UMR 5525. \\ ${ }^{2}$ Koelis SAS, 5. av. du Grand Sablon, 38700 La Tronche, France.
}

\begin{abstract}
The poor signal-to-noise ratio in transrectal ultrasound (TRUS) images makes the fully automatic segmentation of the prostate challenging and most approaches proposed in the literature still lack robustness and accuracy.

However, it is relatively straightforward to obtain high quality segmentations in magnetic resonance (MR) images. In the context of MR to TRUS data fusion the information gathered in the MR images can hence provide a strong prior for US segmentation.

In this paper, we describe a method to non-linearly register a patient specific mesh of the prostate build from MR images to TRUS volume. The MR prior provides shape and volume constraints that are used to guide the MR-to-TRUS surface deformation, in collaboration with a US image contour appearance model. The anatomical point correspondences between the MR and TRUS surfaces are obtained implicitly.

The method was validated on 30 pairs of MR/TRUS patient exams and achieves a mean Dice value 0.85 and a mean surface error of $2.0 \mathrm{~mm}$.
\end{abstract}

Index Terms - US, MR, registration, segmentation

\section{INTRODUCTION}

Prostatic adenocarcimona is the second most common cancer in men. It is the second leading cause of cancer death among men in the US [1]. Currently TRUS imaging is the most accessible and practical modality for guiding needles during diagnostic intervention such as prostate biopsy or therapeutic interventions such as brachytherapy. However, TRUS imaging rarely provides information on the spatial location of prostate cancer and is hence of limited interest for cancer targeting. In recent years, MRI has received increasing interest for localizing prostate cancer. Recent advance in MRI such as MR spectroscopy (MRS), dynamic contrast enhanced MRI (DCE-MRI) with gadolinium injection or lymphotrophic nanoparticle enhanced MRI (LN-MRI) emerge as new promising methods that can improve sensitivity and

*This project has been supported by an ANR grant (PROSPER project) and by the Joseph Fourier University. specificity of cancer detection. MR imaging can now be clinically useful to enhance TRUS imaging and therefore improve needle guidance during biopsy or brachyteraphy. However, the fusion of pre-operative MR data and per-operative TRUS data is currently a technical challenge.

Related previous works include rigid MR/TRUS registration methods [2] which are not suitable when large gland deformation occurs between MR and TRUS imaging or octree based contour registration methods [3] which need prior segmentation on both MR and TRUS images. In [4] a patientspecific statistical model of MR-to-TRUS deformation build from simulated data is used for registration. However, the benefit of statistical modeling based on simulated data is not clear. In particular the very complex nature of the boundary conditions of the simulation (rectum, bladder, probe position), their variability between the acquisitions (bladder and rectal filling, patient position) and the uncertainty about the tissue elasticity parameters could cause biases. The method was validated on intra-prostatic landmarks, for which it yields an accuracy of $2.36 \pm 1.24 \mathrm{~mm}$. The accuracy of the ultrasound surface segmentation was not assessed.

In this paper we propose a method to automatically segment the prostate in TRUS images by deforming a patient specific mesh build from MR images to TRUS data. The nonlinear surface deformation estimation is driven by an image appearance model and constrained by shape and volume priors stemming from the MR surface. In contrast to deformation statistics on simulated data [4], shape and volume priors are simple and less biased representations of the real patient anatomy (up to MR segmentation errors). Only in a second step a biomedical model is used to propagate the surface displacements on the whole volume. In this step, the displacements are imposed as constraints/boundary conditions and it is hence not necessary to model the complex organic boundary conditions (see Fig. 1). We will focus in this article on the segmentation part of the algorithm, which we validate by measuring surface registration accuracy on MR/TRUS pairs stemming from 30 different patients. For completeness, we also introduce a simple bio-mechanical model for deformation propagation and give some preliminary visual results. 


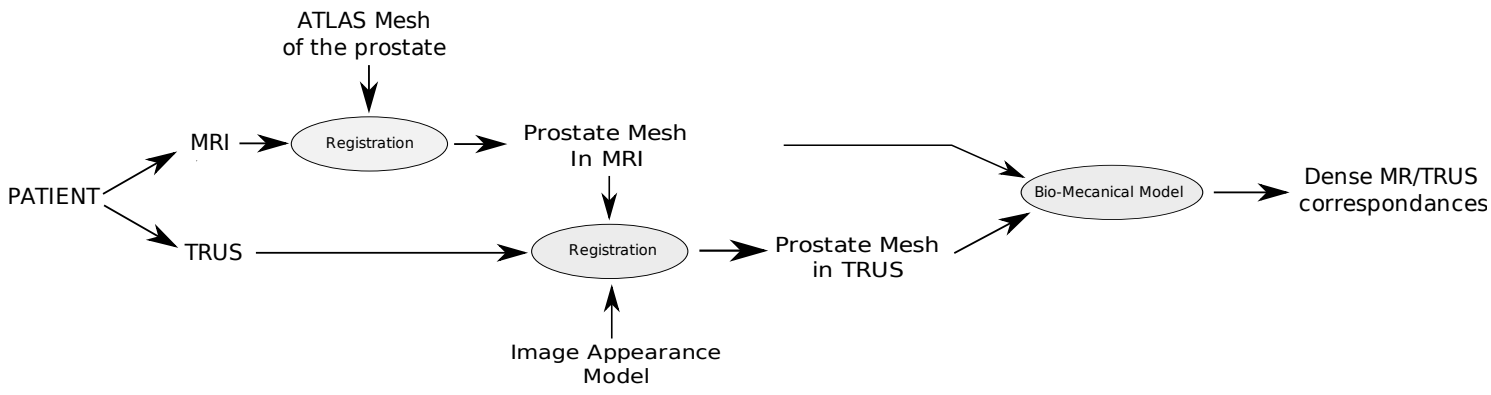

Fig. 1. Overview of the method including MR images segmentation, TRUS images segmentation and MR/TRUS data fusion.

\section{METHOD}

\subsection{MR segmentation}

The first step of the proposed algorithm consists in the segmentation of the MR volume. In this study it is obtained by warping a mean shape of the prostate on a manually segmented point cloud using an interactive approach, but this step can be fully automated [5]. The mean shape stems from the statistical analysis of 23 manual MR segmentations. A patient specific mesh $M$ is hence obtained that can be used as template for US segmentation. $M=\left(M_{1}, \ldots, M_{K}\right)^{t}, M_{i} \in \mathbb{R}^{3}$ is a $K \times 3$ matrix which represents the vertices of the mesh (triangular surface mesh). It is important to note that we thus dispose of an anatomical mapping between the patient-specific mesh and the mean mesh. This makes it possible to construct a spatially varying image appearance model for the prostate capsule in the US volume (see Fig. 1).

\subsection{TRUS Segmentation}

In the following, $T=\left(T_{1}, \ldots, T_{K}\right)^{t}, T_{i} \in \mathbb{R}^{3}$ is a $K \times 3$ matrix that represents the vertices of the deformable TRUS mesh. $T$ is initialized with an approximate repositioning of the MR mesh $M$ on the TRUS images. In a first step, we estimate the rigid body motion of the mesh $T$. In a second step, we rely on a shape-constrained deformable mesh to estimate the residual non-rigid MR/TRUS deformations.

During these two steps, the estimation is driven by the detection of feature points. These detections are based on the minimization of an objective function $E_{i}(k)$ defined for each vertex of the mesh. The feature point $\tilde{T}_{i}=T_{i, \hat{k}}$ is searched along the vertex normal $n_{i}$ :

$$
\hat{k}=\underset{k=-l, \ldots, l}{\operatorname{argmin}} E_{i}(k), \quad T_{i, k}=T_{i}+k h n_{i},
$$

where $l$ defines the length of the search interval, the global parameter $h$ is the profile step size and $k$ is an integer used to explore the profile, i.e. $T_{i, k}$ is a translated position of the vertex $T_{i}$. Different objective functions $E_{i}(k)$ will be defined for rigid and non-rigid registration in the following sections.

\subsubsection{Rigid MR Mesh to TRUS Images Registration}

For rigid registration, an image appearance model is built to represent the statistical variation of the grey-values profiles normal to the mesh surface through each vertex. The Mahalanobis distance from a sample profile to the model mean is then used to locate the feature point :

$$
E_{i}(k)=\left(\bar{g}_{i}-g_{i k}^{\prime}\right) \Sigma_{i}^{-1}\left(\bar{g}_{i}-g_{i k}^{\prime}\right)
$$

Where $g_{i k}^{\prime}$ is the normalized sample profile centered on $V_{i}^{k}$ and oriented in the normal direction $n_{i} . g_{i k}^{\prime}$ is normalized similarly to [6] to take into account global lighting variations across the images. $\bar{g}_{i}$ is the mean normalized grey-level vector of the vertex $i$ and $\Sigma_{i}$ is the associated covariance matrix. A training-set composed of 8 TRUS exams is used to construct the appearance model. Each exam is segmented by warping the mean shape on a dense cloud of manually segmented points on the prostate boundary, i.e. the vertices of the obtained meshes correspond anatomically. This makes it possible to compute the statistics on the intensity profiles.

Starting with a rough repositioning of the MR mesh on TRUS data, we detect at each iteration the feature points and compute the update of vertex positions $T^{t+1}$ by rigidly matching $T^{t}$ on $\tilde{T}^{t}$.

\subsubsection{Non-Rigid MR Mesh to TRUS Images Registration}

Theoretically, due to the incompressible nature of the prostate, the volume of the segmentations should not change significantly between the two modalities. This mechanical property can hence be used as prior to limit the search space of the MR to US surface registration problem. The second prior is the shape of the surface segmented in the MR image, which undergoes only local and limited variations that are mainly caused by US probe pressure. In this section, both priors are injected into an appearance-model driven automatic segmentation to prevent segmentation errors.

Starting from the final estimate of $T$ obtained at the end of the rigid registration, the non-rigid registration is formulated as a coupled minimization of two objective functions with respect to $\mathbf{R}$ and $T$. 
At each iteration $t$, we first estimate the rigid transformation $\mathbf{R}^{t}$ of the template MR mesh $M$ onto the previous TRUS mesh estimation $T^{t-1}$

$$
\mathbf{R}^{t}=\underset{\mathbf{R}}{\operatorname{argmin}} \sum_{i}\left\|T_{i}^{t-1}-\mathbf{R}\left(M_{i}\right)\right\|^{2},
$$

followed by the estimation of $T^{t}$ using volume and shape constraints, and using the detected feature points $\tilde{T}^{t}$ as attractors:

$$
\begin{aligned}
T^{t} & =\underset{T}{\operatorname{argmin}} \mathcal{C}^{t}(T), \text { with } v_{\text {min }}<V(T)<v_{\text {max }} \\
\mathcal{C}^{t}(T) & =\sum_{i}\left(\left\|T_{i}-\tilde{T}_{i}^{t}\right\|^{2}+\frac{\alpha}{N_{i}} \sum_{j \in \mathcal{N}_{i}}\left\|d_{i j}-\mathbf{R}^{t}\left(d_{i j}^{\prime}\right)\right\|^{2}\right),
\end{aligned}
$$

Where $d_{i j}=T_{i}-T_{j}, d_{i j}^{\prime}=M_{i}-M_{j}$ and $V(T)$ is the volume of the mesh $T . \mathcal{N}_{i}$ is the set of neighbors of the vertex $i . N_{i}$ is the number of neighbors of the vertex $i$.

The first term of Eqn. (3) attracts the model towards detected feature points $\tilde{T}_{i}^{t}$. The second term is the shape constraint which ensures that the mesh $T$ stays close to mesh $M \circ \mathbf{R}^{t}{ }^{1}$. The parameter $\alpha$ tunes the strength of the regularization. Finally, the inequality constraint is used to ensure that the prostate volume lies in an acceptable range.

To solve problem 3 , we first need to solve it with equality constraints, i.e. $\min _{T} \mathcal{C}(T)$ subject to $V(T)=v$, where $v$ is the target volume. The Lagrangian of the problem is

$$
\mathcal{L}(T, \lambda)=\mathcal{C}(T)+\lambda \mathcal{S}(T) \quad \text { with } \quad \mathcal{S}(T)=V(T)-v .
$$

Necessary conditions of a minimum of the Lagrangian are

$\partial_{\lambda} \mathcal{L}(T, \lambda)=\mathcal{S}(T)=0$, and

$\partial_{T} \mathcal{L}(T, \lambda)=T-\tilde{T}+\alpha \mathbf{L}(M \circ \mathbf{R}-T)+\lambda \nabla_{T} \mathcal{S}(T)=0$

where $\mathbf{L}$ is a constant $K \times K$ matrix that corresponds to the discretized Laplacian on the meshes ( $T$ and $M$ are topologically identical) and $\lambda$ is the Lagrange multiplier. By linearizing $\mathcal{S}(T)$ around the unconstrained solution denoted $\hat{T}$ we get an expression of the constrained solution that depends on $\lambda$ :

$$
\begin{aligned}
T & =\hat{T}-\lambda(I d-\alpha \mathbf{L})^{-1} \nabla_{T} \mathcal{S}(\hat{T}), \text { with } \\
\hat{T} & =(I d-\alpha \mathbf{L})^{-1}(\tilde{T}-\alpha \mathbf{L} M \circ \mathbf{R}) .
\end{aligned}
$$

The parameter $\lambda$ can be estimated by line search. The algorithm to preserve the volume is summarized in Alg. (1). All systems of linear equations are solved using the conjuate gradient method. The inequality constraint in Eqn. (3) can then be handled by solving the unconstrained problem, searching if a constraint is active and applying corresponding equality constraint if needed.

During the non-rigid registration, the feature point detections are realized by detecting dark-to-bright transitions:

$$
E_{i}(k)=\sum_{i=k+1, \ldots, k+c} I\left(T_{i, k}\right)-\sum_{i=k-c, \ldots, k-1} I\left(T_{i, k}\right)
$$

\footnotetext{
${ }^{1} M \circ \mathbf{R}=\left(\mathbf{R}\left(M_{0}\right), \mathbf{R}\left(M_{1}\right), \ldots, \mathbf{R}\left(M_{K}\right)\right)^{t}$
}

The first term and second term are the sum of the intensity profile values in the outer and inner region respectively. We take into account the $c-1$ first elements in each direction.

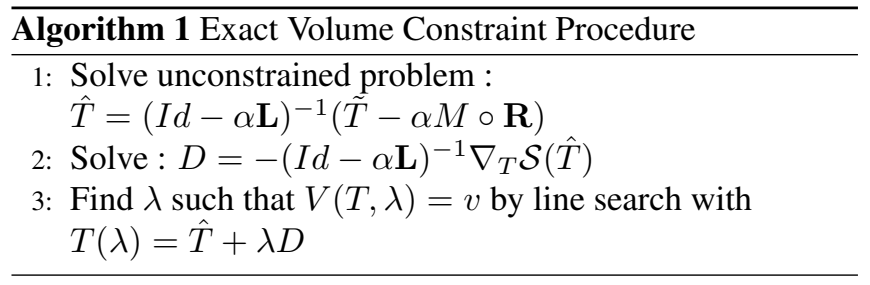

\subsection{MR-US Fusion}

After MR-prior based segmentation of the US volume, the estimated surface deformation is interpolated on the volume of interest $\Omega$. Let us assume that the MR volume is floating and that the US volume is fixed. A natural choice of $\Omega$ is then the TRUS volume. The surface deformations are propagated on $\Omega$ by minimizing the linear elastic energy

$$
\hat{\varphi}=\underset{\varphi}{\operatorname{argmin}} \int_{\Omega} \frac{\mu}{4} \sum_{i=1}^{3}\left\|\nabla\left(\varphi(x)_{i}\right)\right\|^{2}+\frac{\lambda}{2}(\operatorname{div} \varphi(x))^{2} d x,
$$

under the constraints

$$
\varphi\left(T_{i}\right)=\mathbf{R}^{n}\left(M_{i}\right)-T_{i} \forall i,
$$

where $\varphi: \mathbb{R}^{3} \rightarrow \mathbb{R}^{3}$ is the displacement function and $\mathbf{R}^{n}$ is the result of the final iteration of Eqn. (2). Eqn. (6) is solved by gradient descent using the semi-implicit diffusion scheme

$$
\frac{\varphi^{t+1}-\varphi^{t}}{\delta}+\mu \Delta \varphi+(\lambda+\mu) \nabla \operatorname{div} \varphi=0,
$$

where $t \in \mathbb{N}$ is the iteration number and $\delta \in \mathbb{R}$ is the discretization granularity of the partial differential system. The displacement constraints (7) are enforced at each iteration. Eqn. (8) is solved using the full multigrid strategy and GaussSeidel relaxation for all $x \in \Omega$ that lie on a regular, isotropic 3D grid, which defines the spatial discretization of the deformation function. Since the constraints do in general not lie on a node of the regular grid it is necessary to project them onto their 8 nearest grid points using tri-linear distance weighting. After processing all constraints, the accumulated weighted distances at each grid point are normalized with the corresponding accumulated weights (if not zero).

\section{EXPERIMENTS AND VALIDATION}

All MR exams are acquired with a 1.5 Tesla (T) scanner using a surface coil. Ultrasound (US) images are obtained using a 3D ultrasound device and a transrectal probe. The validation has been carried out using a data-set composed of $30 \mathrm{cou}-$ ples of MR/TRUS exams. 8 additional TRUS exams were used to build the appearance model. The accuracy of the 


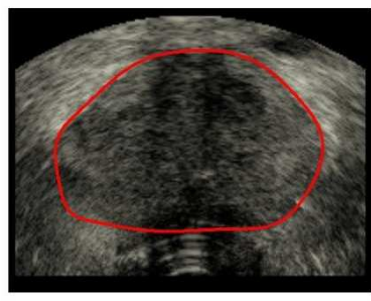

A)

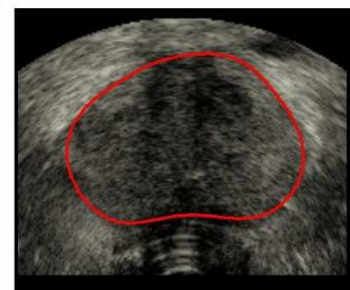

B)

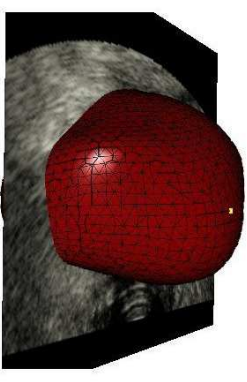

C)

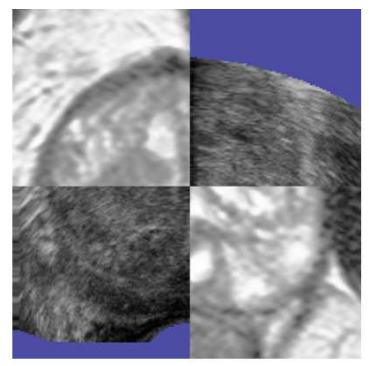

D)

Fig. 2. Final contours on TRUS images compared with manual segmentation. A: automatic segmentation. B: manual segmentation. C: final deformable mesh. D: MR image data superimposed on TRUS image data.

Table 1. Segmentation accuracy. PPV: Positive Predictive Value, Sens: Sensitivity, mean err: mean value of surface errors, RMSD: Root mean square deviation of surface errors. Surface errors are distances between each vertex of the automatic segmentation and its closest point on the manual segmentation.

\begin{tabular}{ccccc}
\hline PPV & Sens & Dice & mean err. & RMSD \\
\hline 0.82 & 0.88 & 0.85 & $2.0 \mathrm{~mm}$ & $1.5 \mathrm{~mm}$ \\
\hline
\end{tabular}

prostate segmentation in TRUS images has been validated by comparing manual segmentations to the automatic segmentations. The manual segmentations were obtained by accurately identifying points on the prostate boundaries and by reconstructing the prostate using a deformable surface which does not use any prior information on the prostate shape. The maximum and minimum volume used in Eqn. (3) are determined using the following formula: $v_{\max }=(1+p) V_{M R}$ and $v_{\min }=(1-p) V_{M R}$. Where $V_{M R}$ is the prostate volume obtained in MRI. The parameter $p$ was set to $10 \%$, which corresponds approximatively to the volume fluctuations that we could observe. The segmentation accuracy has been evaluated using surface-based and volume-based measures. Results are reported in table 1. Fig. 2. presents an example of MR segmentation and the corresponding automatic segmentation in TRUS and shows an example of MR/TRUS fusion where the MR image is superimposed on the TRUS images.

\section{DISCUSSION \& CONCLUSION}

In this paper, we have proposed an automatic prostate segmentation method in TRUS images based on the non-rigid registration of a patient specific mesh obtained from MR segmentation. MR-to-TRUS mapping is performed by propagating surface displacements in the whole volume. The presented approach, validated on 30 couples of MR/TRUS exams, is robust and yields more accurate segmentations of the prostate than methods that do not use MR priors.
A remaining issue is that poor initial manual positioning of the MR template can lead to false anatomical mappings. A careful initialization is hence necessary. This problem could be attenuated if the deformation propagation algorithm would use vertex to surface distances as constraints instead of the distances between corresponding vertices. We are currently implementing this feature.

\section{REFERENCES}

[1] A. Jemal, R. Siegel, E. Ward, T. Murray, J. Xu, and M.J. Thun, "Cancer statistics, 2007," CA: a cancer journal for clinicians, vol. 57, no. 1, pp. 43, 2007.

[2] S. Xu, J. Kruecker, B. Turkbey, N. Glossop, A.K. Singh, P. Choyke, P. Pinto, and B.J. Wood, "Real-time MRITRUS fusion for guidance of targeted prostate biopsies," Computer aided surgery: official journal of the International Society for Computer Aided Surgery, vol. 13, no. 5, pp. 255, 2008.

[3] C. Reynier, J. Troccaz, P. Fourneret, A. Dusserre, C. Gay-Jeune, J.L. Descotes, M. Bolla, and J.Y. Giraud, "MRI/TRUS data fusion for prostate brachytherapy. Preliminary results," Medical Physics, vol. 31, pp. 1568, 2004.

[4] Yipeng Hu, Hashim Uddin Ahmed, Clare Allen, Doug Pendsé, Mahua Sahu, Mark Emberton, David Hawkes, and Dean Barratt1, "MR to Ultrasound Image Registration for Guiding Prostate Biopsy and Interventions," MICCAI, vol. 1, pp. 787-794, 2009.

[5] S. Martin, V. Daanen, and J. Troccaz, "Automated segmentation of the prostate in $3 \mathrm{~d} \mathrm{mr}$ images using a probabilistic atlas and a spatially constrained deformable model," Medical Physics, vol. 37, no. 3, 2010.

[6] T.F. Cootes, C.J. Taylor, D.H. Cooper, J. Graham, et al., "Active shape models-their training and application," Computer vision and image understanding, vol. 61, no. 1, pp. 38-59, 1995. 